\title{
A Variation of Flexor Carpi Ulnaris Muscle: A Case Report
}

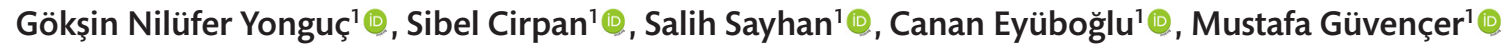 \\ 'Dokuz Eylül University School of Medicine, Department of Anatomy, İzmir, Turkey \\ Address for Correspondence: Mustafa Güvençer, E-mail: mustafa.guvencer@deu.edu.tr \\ Received: 11. 27.2017; Accepted: 12.18.2017; Available Online Date: 30.05.2018 \\ (C) Copyright 2018 by Dokuz Eylül University, Institute of Health Sciences - Available online at www.jbachs.org \\ Cite this article as: Yonguç GN, Cirpan S, Sayhan S, Eyüboğlu C, Güvençer M. A Variation of Flexor Carpi Ulnaris Muscle: A Case Report. J Basic Clin Health Sci 2018; 2:57-59. https://doi. \\ org/10.30621/jbachs.2018.320
}

\begin{abstract}
Purpose: Flexor carpi ulnaris muscle (FCUM) is one of the muscles of the superficial flexor group which is located most medially in the forearm. This study aimed to emphasize the importance of variations of the FCUM in clinical diagnosis and surgical procedures.

Methods: While performing routine gross anatomy dissection of an adult male formaldehyde fixed cadaver in laboratory of Anatomy Department, we observed a unilateral variation of FCUM in the left upper extremity.

Results: FCUM was originated with one common tendon and separated into two bellies at the distal half of forearm, and extended with two tendons. The connecting tendons were inserted to pisiform, hamate bones, and proximal of the fifth metacarpal bones.

Conclusion: The detection of FCUM variations is significantly important not only in the treatment of pressure related symptoms which they cause but also in reconstructive and orthopedic surgery.

Keywords: Flexor carpi ulnaris muscle, variation, forearm
\end{abstract}

\section{INTRODUCTION}

Flexor carpi ulnaris muscle (FCUM) is an important muscle in the forearm belonging to the anterior flexor compartment, and it provides strength to wrist flexion and ulnar adduction. Besides, it is used for flap surgery (1).

The prevalence of anomalies related to FCUM is rare. No anomalies of the FCUM were found among 5000 cadaver specimens which were surveyed (1). However, a number of reported FCUM anomalies have been established via ultrasound, during surgery, or cadaver dissection $(2,3)$. Therefore, the knowledge of possible major anomalies which may give rise to misidentification of anatomical structures during surgical procedures on the forearm and wrist is of crucial importance (4).

\section{CASE REPORT}

A variation of FCUM on the left forearm of an adult male cadaver was observed during anatomical dissection. The cadaver belonging to the Anatomy Laboratory of Dokuz Eylul University, School of Medicine was fixed with \%10 formalin solution.

The anomalous FCUM was arising with the common tendon from the medial epicondyle; however, the belly of the muscle was split into two parts proximally at the forearm, and extended with two tendons through the distal forearm (Figure 1). The fibers of these tendons were attached to the pisiform, the hamate and proximal of the fifth metacarpal bone anterior to flexor retinaculum (Fig. 2). Ulnar nerve and artery were located lateral to the tendon of the variant muscle (Figure 2). We did not observe any other variations on the left upper extremity of the cadaver. This case was examined morphologically, and photographed.

\section{DISCUSSION}

The flexor carpi ulnaris muscle (FCUM), positioned most medially in the forearm, belongs to the superficial flexor group of muscles (5). FCUM is a genuinely bipennate muscle and possesses an independent nerve and has its own vascular supply (6). Its humeral head is small, and originates from the medial epicondyle; the origin of the ulnar head is extensive, and it attaches to the medial margin of the olecranon and proximal two-thirds of the posterior border of the ulna. At its anterolateral border, in its distal half, it forms a thick tendon which is attached to the pisiform, and extends to the hamate and fifth metacarpal (5).

Soft tissue defects of the posterior elbow may develop secondary to trauma and wound dehiscence due to burns, chronic 

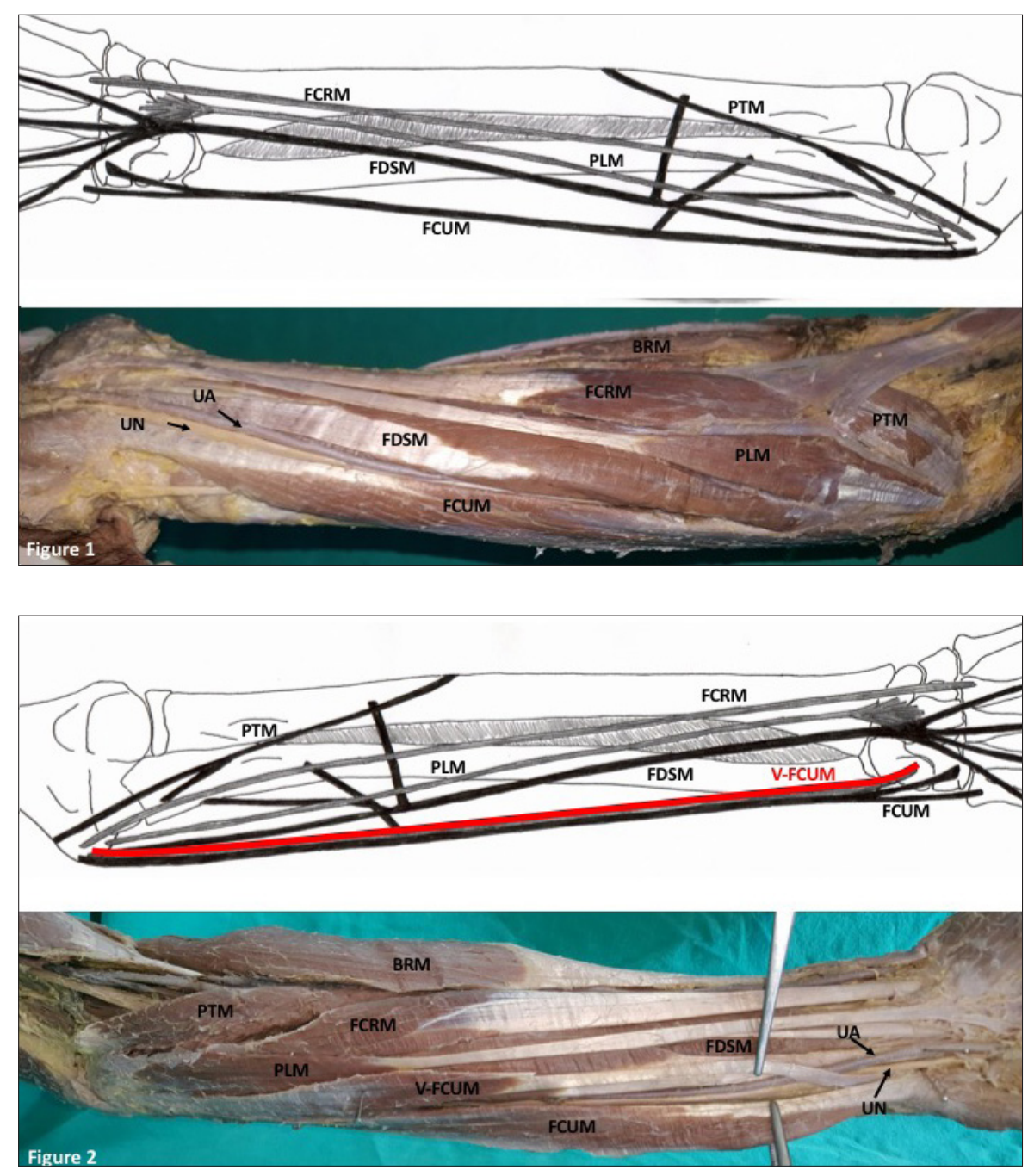

Figure 1. Normal placement of the forearm muscles in the upper right extremity (BRM: Brachioradialis muscle, FDSM: Flexor digitorum superficialis muscle, PTM: Pronator teres muscle, FCRM: Flexor carpi radialis muscle, PLM: Palmaris longus muscle, FCUM: Flexor carpi ulnaris muscle, UA: Ulnar artery, UN: Ulnar nerve). inflammation, and bursitis (7-9). The larger ulnar and smaller humeral heads comprising the FCUM are both able to affect independently with one being confined for wrist flexion and ulnar deviation strength while the other is for tendon transfer or soft tissue coverage of the region $(10,11)$.

The rare anatomic variations which exist emphasize the importance of appropriate preoperative planning, and the need for the consideration of alternative reconstructive options (12).

These anomalies may be clinically asymptomatic, and may be detected incidentally during anatomical dissections or surgical treatment of lacerations (13). They may rarely be accompanied with pain, mass in forearm, or with symptoms of ulnar nerve compression (2). Bhardwaj et al. (13) propose a system for the classification of FCUM variations based on reviews of literature, and they define three major groups of anomalies:

Type 1: Single muscle with two tendons (split tendon): the presence of a single muscle with two separate tendons.

Type 2: Each head forming separate muscle and tendon (Digastric FCU): the presence of two heads each forming a separate muscle and tendon.
Type 3: Extra muscle in addition to normal FCUM (accessory FCU): the presence of an extra muscle in alongside the normal FCUM

Ciftcioglu et al. (14) reported a FCUM that possessed only a humeral head; another muscle belly originating from medial epicondyle lying between flexor digitorum superficialis and FCUM and was attached to triquetral, hamate bones, and flexor retinaculum.

Sawant et al. (15) reported a FCUM which had separate humeral and ulnar heads, and combined tendons just before their insertion to pisiform. In both of these studies the muscular anomalies observed were classified as type 2 described by Bhardwaj et al. (13).

We observed the anomalous FCUM which has a separate muscle belly extending from the proximal part of the forearm, and form a separate tendon inserting to the to the pisiform, the hamate and proximal of the fifth metacarpal bone. We interpreted it as type 2 (Fig. 1 and 2) according to classification described by Bhardwaj et al. (13).

The detection of FCUM variations is significantly important not only in the treatment of pressure related symptoms which they cause but also in reconstructive and orthopedic surgery. 
Informed Consent: Written informed consent was obtained from patient who participated in this case

Peer-review: Externally peer-reviewed.

Author Contributions: Concept - MG; Design - MG; Supervision - MG, GNY; Literature Search - GNY, SC, SS, CE; Writing - GNY, SC; Critical Reviews - GNY, MG; Cadaver Dissection - GNY, SC, SS, CE

Conflict of Interest: The authors declare that there are no conflicts of interest.

Financial Disclosure: This study has received no financial support

\section{REFERENCES}

1. Ang GG, Rozen WM, Vally F, Eizenberg N, Grinsell D. Anomalies of the flexor carpi ulnaris: clinical case report and cadaveric study. Clin Anat 2010;23:427-430. [CrossRef]

2. Georgiev GP, Jelev L, Ovtscharoff WA. Unusual combination of muscular and arterial variations in the upper extremity: a case report of a variant palmaris longus and an additional tendinous portion of the flexor carpi ulnaris together with a persistent median artery. Anatomy 2009;3:58-61.

3. O'Hara J, Stone JH. Ulnar neuropathy at the wrist associated with aberrant flexor carpi ulnaris insertion. J Hand Surg Am 1988;13:370372

4. Alvin M, Alan N, Leone J, Fredieu JR. A unilateral accessory flexor carpi ulnaris muscle observed during cadaveric dissection. Clin Anat 2011;24:971-973. [CrossRef]

5. Standring S. Elbow, Chapter 51. In: Standring S, editor. Gray's Anatomy, 39th ed., London, England: Churchill Livingstone Elsevier; 2005. p.877.

6. Lim AY, Kumar VP, Hua J, Pereira BP, Pho RW. The neuromuscular compartments of the flexor carpi ulnaris. Plast Reconstr Surg 1999;103:1046-1051.

7. Meals RA. The use of a flexor carpi ulnaris muscle flap in the treatment of an infected nonunion of the proximal ulna. A case report. Clin Orthop Relat Res 1989;(240):168-172.
8. Culbertson $\mathrm{JH}$, Mutimer $\mathrm{K}$. The reverse lateral upper arm flap for elbow coverage. Ann Plast Surg 1987;18:62-68.

9. Raddatz DA, Hoffman GS, Franck WA. Septic bursitis: presentation, treatment and prognosis. J Rheumatol 1987;14:1160-1163.

10. Lim AY, Kumar VP, Pereira BP, Hua J. Independent function in a tendon transfer of the split flexor carpi ulnaris. Plast Reconstr Surg 1999;104:1739-1741.

11. Lingaraj K, Lim AY, Puhaindran ME, Kumar PV. Case report: the split flexor carpi ulnaris as a local muscle flap. Clin Orthop Relat Res 2007;455:262-266. [CrossRef

12. Sharpe F, Barry P, Lin SD, Stevanovic M. Anatomic study of the flexor carpi ulnaris muscle and its application to soft tissue coverage of the elbow with clinical correlation. J Shoulder Elbow Surg 2014;23:82-90. [CrossRef]

13. Bhardwaj P, Bhandari L, Sabapathy SR. Supernumerary flexor carpi ulnaris -case report and review. Hand Surg 2013;18:393-397. [CrossRef]

14. Cıftçıoğlu E, Kopuz C, Corumlu U, Demır MT. Accessory muscle in the forearm: a clinical and embryological approach, Anat Cell Biol 2011;44:160-163. [CrossRef]

15. Sawant SP, Shaikh ST, More RM. Variant flexor carpi ulnaris muscle and variant course of ulnar artery in forearm. Int J Mod Eng Res 2012;2:4108-4110. 